\title{
Mobilität 4.0: Deutschlands erste autonome Buslinie in Bad Birnbach als Pionierleistung für neue Verkehrskonzepte
}

\author{
Michael Barillère-Scholz, Chris Büttner und Andreas Becker
}

Die Autoren geben einen Einblick in die Überlegungen zur Inbetriebnahme eines autonomen Shuttlebusses in Bad Birnbach. Dargelegt werden die Schwierigkeiten und Hürden, die aus rechtlicher Sicht genommen werden mussten, um den autonomen Shuttlebus tatsächlich auf die Straße zu bringen. Es zeigt sich, dass autonome Fahrzeuge im ÖPNV einer der Meilensteine der nächsten Jahre sein und den Mobilitätsmarkt von morgen prägen werden.

\subsection{Mobilitätsmarkt von morgen}

Der Öffentliche Personennahverkehr (ÖPNV) in Deutschland steht vor großen Herausforderungen: In den Städten nimmt der Verkehr mehr und mehr zu - Staus, Umweltverschmutzung und Parkplatzprobleme sind die Folge. Zur Bewältigung dieser Herausforderungen muss der Öffentliche Verkehr (ÖV) gestärkt werden und sich zugleich angebotsseitig an den individuellen Bedürfnissen der Menschen orientieren, um so attraktiver zu werden (Horn et al. 2018). Neue Mobilitätsdienstleister erweitern in den Städten das Mobilitätsangebot, indem sie bereits heute in zahlreichen Städten bedarfsgerechte, individualisierte Verkehrsangebote, sogenannte On-Demand Mobilität, anbieten (Buffat et al. 2018). Studien zeigen jedoch, dass diese häufig zu mehr Verkehr in den Städten führen und den ÖV substituieren (Schaller 2017). Gleichzeitig verlassen immer mehr Menschen den ländlichen Raum oder entscheiden sich aktiv gegen den ÖV und für den privaten

M. Barillère-Scholz C C. Büttner $(\bowtie) \cdot A$. Becker ioki GmbH, Frankfurt am Main, Deutschland

E-Mail: michael.barillere-scholz@ioki.com; chris.buettner@ioki.com;

andreas.becker@ioki.com 
Pkw, weil es für die sog. Erste und Letzte Meile kein passendes Angebot gibt (Herget et al. 2018; Conrad 2016).

Neue Mobilitätsangebote verändern den Markt grundlegend - die Unabhängigkeit vom eigenen Auto und nachhaltige Fortbewegung treten zunehmend in den Vordergrund. Mobility as a Service, alternative Antriebstechniken und autonomes Fahren sind dabei wichtige Treiber der Mobilität der Zukunft (ADAC 2017; Berylls 2017; Pavone 2015). Digitalisierung spielt diesbezüglich eine entscheidende Rolle - sie ist bereits jetzt ein fester Bestandteil des täglichen Lebens und bietet wichtige Chancen für nachhaltige Mobilität (Münchner Kreis 2017). Deutschland darf sich vor diesen Entwicklungen nicht verschließen. Die Mobilität befindet sich im Wandel und wird sich bis 2025 deutlich verändern. Niedrigpreis-Mobilität und Sharing-Angebote werden fest etabliert sein - sowohl im ländlichen als auch im städtischen Raum. Digitalisierung prägt den Mobilitätsmarkt von morgen: Apps geben volle Transparenz über bestehende Angebote und Preise. Digitale Reisebegleiter beeinflussen die Verkehrsmittelwahl. Erste Prototypen von autonomen Fahrzeugen finden ihren Platz auf der Straße und ermöglichen flexible und individuelle Mobilität - vollautomatisiert. Autonome Verkehrsangebote werden den Mobilitätsmarkt der Zukunft grundlegend verändern (Pavone 2015). Großteile des Motorisierten Individualverkehrs (MIV) und des ÖV werden zu einem neuen Verkehrsmarkt, dem Individuellen Öffentlichen Verkehr (IÖV) verschmelzen (vgl. Abb. 2.1) (acatech Studie 2016).

Der IÖV ist intelligent-einfach organisiert. Tür-zu-Tür-Verkehre mit geringer Wartezeit können sowohl in der Stadt als auch im ländlichen Raum zu ÖV-Preisen wirtschaftlich angeboten werden. Dabei kommen unterschiedliche Gefäßgrößen zum Einsatz. Somit verliert das eigene Fahrzeug an Bedeutung. Der IÖV ersetzt zunehmend das eigene Auto und ergänzt sinnvoll den klassischen, liniengebundenen ÖV. Das neue System unterscheidet sich vom klassischen ÖPNV insofern, als dass die heute noch üblichen festen Fahrpläne und festen Fahrwege an Bedeutung verlieren und Mobilitätsangebote zunehmend ,on demand“ - also auf Kundenanforderung angepasst - bereitgestellt werden. Fahrgäste und Mobilitätsanbieter werden über neue Marktplätze in Form digitaler Plattformen
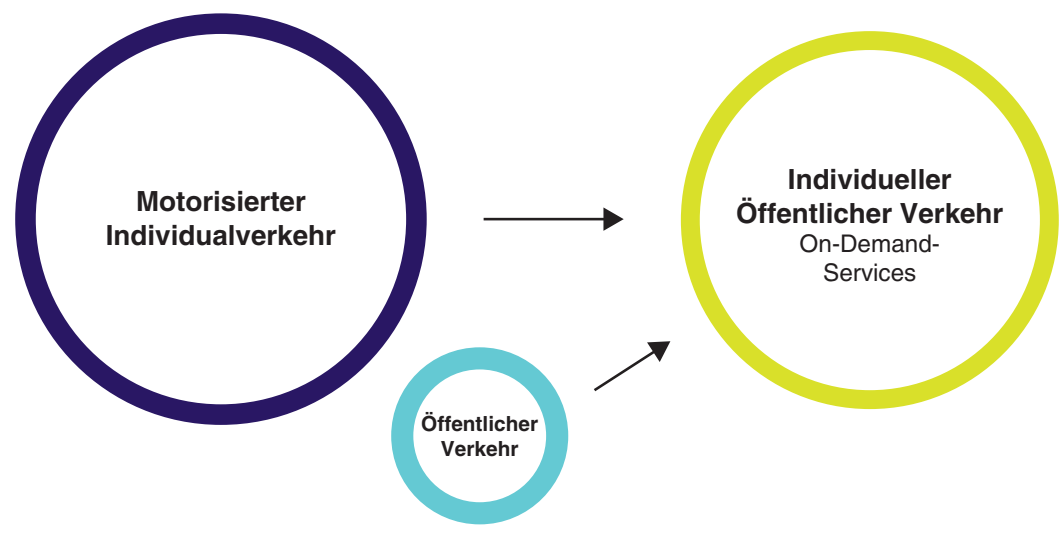

Abb. 2.1 Wandel der Struktur des Mobilitätsmarktes 
interagieren. So schafft die autonome On-Demand-Mobilität der Zukunft nicht nur persönliche Freiräume, sondern wirkt sich auch auf die Lebensqualität der Menschen in der Stadt und auf dem Land dauerhaft positiv aus (Hunsicker et al. 2017). Autonome Fahrzeuge werden kommen. Technologische und regulatorische Hürden werden genommen. Es ist davon auszugehen, dass die Nutzung autonomer Fahrzeuge eine schnelle Verbreitung finden wird, da der gesellschaftliche und individuelle Nutzen groß ist (VDV 2017; acatech Studie 2016). Das gemeinsame Credo für die Mobilität der Zukunft wird sein: Mehr Mobilität, weniger Verkehr.

\section{2 ioki als Pionier für innovative Mobilitätslösungen}

ioki sieht großes Potenzial darin, den ÖV nachhaltiger und effizienter zu gestalten und individuelle Mobilität ohne eigenes Auto möglich zu machen. Dabei muss der Anspruch sein, Mobilität für alle zu ermöglichen: sowohl in der Stadt als auch auf dem Land. Als Komplettdienstleister für digitale Mobilitätsformen auf der Straße entwickelt ioki zukunftsfähige Lösungen, die Mobilität langfristig sichern, wirtschaftlich tragfähig und sozial ausgewogen sind, gleichzeitig die Umwelt schonen und somit die Lebensqualität erhöhen. Als Geschäftszweig der Deutschen Bahn AG ist ioki Teil der Digitalisierungsoffensive und der festen Überzeugung, dass ein gesundes Ökosystem gut integrierte und keine sich kannibalisierenden und damit ineffizienten Mobilitäts-Services braucht. Dabei darf der Blick auf den Endkunden sowie die voranschreitende Digitalisierung nicht verloren werden: Um Kundenbedürfnisse bestmöglich erfüllen zu können, müssen sowohl die Mobilität auf dem Land neu gedacht und gestaltet als auch digitale Mobilitätsservices sinnvoll in Städte integriert werden.

ioki gestaltet mit Leidenschaft die Mobilität von morgen - flexibel, individuell, auf Abruf und für jeden überall und jederzeit verfügbar. Die 100-prozentige DB-Tochter bietet smarte, maßgeschneiderte On-Demand-Lösungen. Mit Hilfe von Big-Data-Analysetools werden ökonomisch und ökologisch sinnvolle Einsatzgebiete unter Berücksichtigung bestehender Verkehrsangebote mit besonderem Fokus auf den ÖPNV identifiziert und On-Demand-Angebote in die lokale Mobilität integriert. Die ioki-Plattform bietet Bausteine für die Einführung und den Betrieb digitaler On-Demand-Verkehre. Der selbst entwickelte Algorithmus stellt ein intelligentes Pooling und Routing und eine effiziente Auslastung der Strecken und Fahrzeuge sicher. Als Brücke zur intelligenten Mobilität auf der Straße ermöglicht es ioki Dritten, wie z. B. Städten, Verkehrsunternehmen und -verbünden, kommunalen Aufgabenträgern und Firmen, bedarfsgerechte und moderne Mobilitätskonzepte für ihre Kunden anzubieten und damit Mobilität - auch automatisiert und perspektivisch vollautonom - neu zu denken. 


\subsection{Mobilität von morgen heute gedacht: Testfelder zum autonomen Fahren im Öffentlichen Verkehr}

ioki entwickelt seine Lösungen zukunftsorientiert: Testfelder zum autonomen Fahren im ÖV stellen sicher, dass die datengestützte Verkehrsanalyse und die Plattformlösungen sowie der operative Betrieb von On-Demand-Mobilitätsangeboten schon heute bereit sind für die autonome Mobilität von morgen. In Zukunft werden On-Demand-Verkehre - auch ohne Fahrer - Menschen auf Wunsch von A nach B befördern (Deutsche Bahn 2018; VDV 2017; acatech Studie 2016). Dazu müssen perspektivisch Systembausteine bereitgestellt werden, um es Kunden wie Verkehrsanbietern, Gemeinden und Städten zu ermöglichen, neue Mobilitätsformen im Öffentlichen Verkehr zu betreiben. Im Rahmen von Testfeldern werden autonome Technologien in ausgewählten Anwendungsfällen frühzeitig in der Praxis eingesetzt und neue Verkehrsangebote geschaffen, sowie ganzheitliche Fahrzeugkonzepte entwickelt (s. Abb. 2.2) (acatech Studie 2016).

Bereits heute setzt ioki autonomes Fahren im öffentlichen Linienverkehr ein: Gemeinsam mit dem Landkreis Rottal-Inn und DB Regio sowie in enger Zusammenarbeit mit dem Fahrzeughersteller EasyMile, dem TÜV Süd und der Marktgemeinde Bad Birnbach hat ioki den ersten autonomen Linienverkehr Deutschlands auf die Straße gebracht (vgl. Abb. 2.3). Mit bayerischen Hochschulen wurde eine Forschungspartnerschaft geschlossen, um das Testfeld wissenschaftlich zu begleiten. Die Erkenntnisse der unterschiedlichen Forschungsvorhaben sind Gegenstand dieser Publikation.

Mit dem Pilotprojekt in Bad Birnbach soll bewiesen werden, dass ein dauerhafter Betrieb eines autonom fahrenden Prototyps im ländlichen Raum schon heute realisiert werden kann. Die Ziele des Projekts leiten sich aus der Vision ab, einen autonomen LetzteMeile-Dienst im ländlichen Raum zu etablieren und diesen in den ÖPNV zu integrieren (Bad Birnbach 2018):

- Etablierung eines autonomen Linienbetriebs zwischen der Rottal-Terme in Bad Birnbach und dem Stadtzentrum des Kurorts

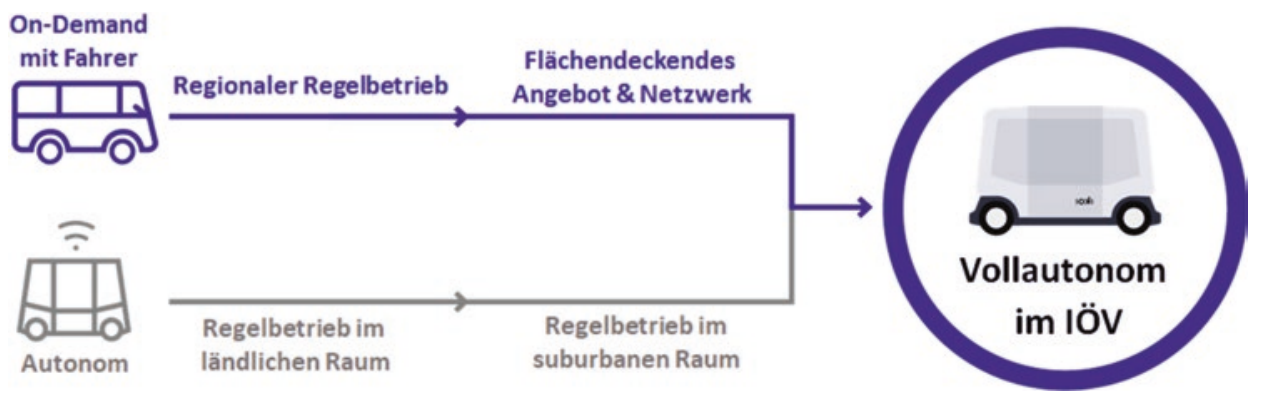

Abb. 2.2 Evolution vollautonomer Mobilitätsangebote im IÖV 


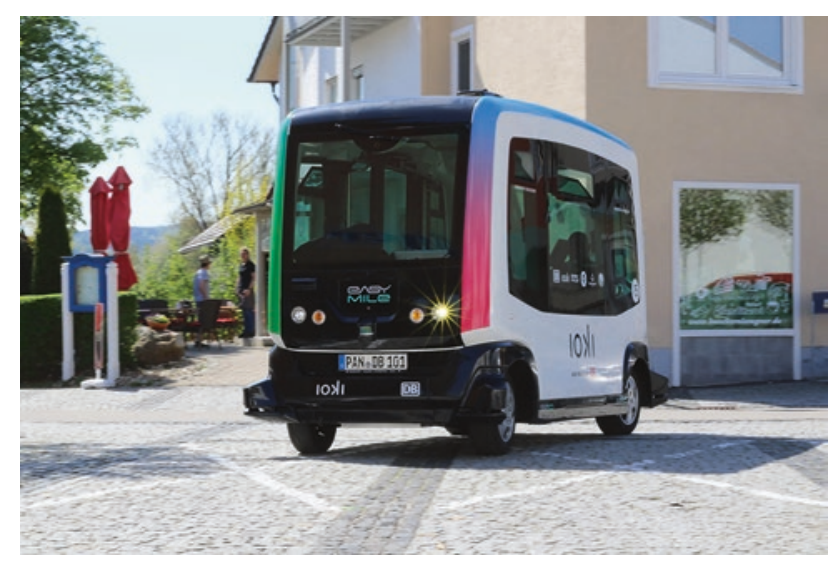

Abb. 2.3 Automatisierter Shuttlebus in Bad Birnbach

- Anschluss der autonom gefahrenen Strecke an den Hochleistungs-ÖV mit Anbindung an den Bahnhof Bad Birnbach

- Forschung und Weiterentwicklung zum Thema Nutzerakzeptanz von autonomen Fahrzeugen auf öffentlicher Straße

Die erste Ausbaustufe des Projekts wurde am 25. Oktober 2017 gestartet. Die Erlaubnis zur Teilnahme am Straßenverkehr nach $§ 29$ Abs. 3 Satz 2 StVO wurde vom Landratsamt Rottal-Inn erteilt. Die ursprüngliche Strecke führt vom Ortszentrum zur Therme des Kurorts. Zum Start des autonomen Betriebs mussten nur geringe infrastrukturelle Maßnahmen in Bad Birnbach getätigt werden. So ist beispielsweise die Streckenbreite im Bereich des Ortszentrums leicht vergrößert worden, um einen ordnungsgemäßen und flüssigen Betrieb gewährleisten zu können (Bad Birnbach 2018).

Der Hersteller und die Betreiberschaft verfolgen hierbei die Philosophie, dass Anpassungen der Infrastruktur gering ausfallen sollten, um hohe Zusatzkosten zu vermeiden. Im ersten Jahr seit der Premiere im Oktober 2017 hat das Fahrzeug mehr als 15.000 Kilometer autonom zurückgelegt und mehr als 25.000 Fahrgäste befördert (ioki 2019).

Im August 2018 folgte in der zweiten Ausbaustufe des Projekts eine Streckenerweiterung mit einer Verdopplung der gefahrenen Strecke von 700 Meter auf 1400 Meter (Deutsche Bahn 2018). Zum Set-up der neuen Strecke wurden weitere geringe infrastrukturelle Maßnahmen durchgeführt. Dies betraf beispielsweise die Installation sogenannter Lokalisierungspaneele, die der Orientierung auf dem freien Feld dienen und für die Positionsbestimmung des autonomen Busses essenziell sind.

In der zweiten Ausbaustufe wurde der Anschluss an den Hochleistungs-ÖV mit der Erschließung der Strecke zum Bahnhof Bad Birnbach umgesetzt, welcher ca. 1,8 Kilometer vom Ortszentrum entfernt ist. Die Erschließung des Bahnhofs gestaltet sich aus Projektsicht als verkehrstechnisch hoch komplex, da die Route des autonomen Kleinbusses zum 
Bahnhof über eine Landstraße führt. Dies beinhaltet eine stärkere Interaktion mit anderen Verkehrsteilnehmern sowie Höchstgeschwindigkeiten von bis zu $60 \mathrm{~km} / \mathrm{h}$.

Betrieben wird der Einsatz des autonomen Kleinbusses von der DB-Tochter DB Regio Bus Ostbayern. Die Regio Bus Ostbayern stellt hierbei auch den Betriebsleiter nach der Verordnung über den Betrieb von Kraftfahrunternehmen im Personenverkehr (BOKraft), koordiniert das erforderliche Sicherheitspersonal und kümmert sich um Wartungs- sowie Reparaturarbeiten an den Kleinbussen. Die Projektorganisation und -koordination sowie die Weiterentwicklung des Projekts läuft unter der Federführung von ioki.

\subsection{Technische Details des Einsatzfahrzeugs}

In Bad Birnbach kommt das Fahrzeug Easymile EZ10 zum Einsatz, ein elektrisches Shuttle des Start-ups EasyMile, das Software für den Betrieb autonomer Fahrzeuge entwickelt. Die Fahrzeugplattform selbst wird vom französischen Hersteller Ligier hergestellt. Das Shuttle folgt - wie auf virtuellen Schienen - automatisiert der Route, die inklusive Haltestellen einmalig per Laserscanner in den Bordcomputer eingelesen wurde (Deutsche Bahn 2019). Insgesamt verfügt es über ein voll funktionsfähiges autonomes Fahrsystem, welches aus mehreren Sensoren besteht. Hierzu zählen insgesamt sieben LiDAR-Sensoren, GPS, Odometrie sowie Inertialsensoren (Easymile 2019).

Das EZ10 ist ein batteriebetriebenes Elektrofahrzeug (BEV), welches nicht nur leise und umweltfreundlich ist, sondern auch weder Lenkrad noch Gas- und Bremspedal besitzt. An Bord ist immer ein Fahrtbegleiter (sog. Safety Operator), der bei Bedarf ins Fahrgeschehen eingreift, beispielsweise durch das Auslösen eines sofortigen Stopps oder das manuelle Umfahren von Hindernissen per Joystick (Deutsche Bahn 2019).

Der Antriebsstrang des Fahrzeugs basiert auf einer 48-Volt-Bordarchitektur. Die Kapazität der eingebauten Lithium-Ionen-Batterien erlaubt eine maximale Einsatzdauer von 14 Stunden (Easymile 2019). Die Geschwindigkeit des autonomen Kleinbusses ist bisher auf maximal 15 Stundenkilometer begrenzt. Dank einer zusätzlichen Rampe können auch mobilitätseingeschränkte Personen oder Fahrgäste mit Kinderwagen problemlos ein- und aussteigen (Bad Birnbach 2018).

Sicherheit im autonomen Betrieb steht für alle Beteiligten des Projekts an oberster Stelle. So wurden durch den Projektpartner TÜV Süd, der auf dem Gebiet des autonomen Fahrens umfassende Expertise besitzt, umfangreiche Tests am Fahrzeug durchgeführt. Hierbei wurden die Fahreigenschaften des Fahrzeugs in dynamischen Tests sowie das Sicherheits- und Betriebskonzept des Busses begutachtet und geprüft (Heise 2018; Deutsche Bahn 2018).

Als erstes Unternehmen mit einer Zulassung für einen autonomen Verkehr im öffentlichen Raum setzt ioki alles daran, auch zukünftig mit großen Schritten voranzugehen. Derzeit gibt es noch keine standardisierten Verfahren zur Zulassung autonomer Verkehrsangebote (Heise 2018). Aus diesem Grund arbeitet ein interdisziplinäres Team aus IT- und Verkehrsexperten sowie Softwareexperten und -entwicklern gemeinsam mit Partnern aus 
Industrie, Wissenschaft und Politik an der Weiterentwicklung dieser Fahrzeugsysteme sowie an den regulatorischen Grundlagen zur Einführung eines fahrerlosen On-DemandMobilitätskonzepts.

\subsection{Lerneffekte des autonomen Betriebs sowie Ausblick auf zukünftige Projekte}

Aus dem derzeit 14-monatigen Einsatz (Stand: Dezember 2018) konnten vielfältige Erfahrungen bezüglich der Handhabung des technischen Systems sowie der Nutzerakzeptanz des autonomen Fahrzeugs gewonnen werden, insbesondere in Bezug auf die Akzeptanz älterer Nutzergruppen auf dem Land. Dem verkehrlichen Nutzen solcher Systeme kommt hier eine besondere Bedeutung zu. Die gewonnenen Erkenntnisse dienen hierbei als Indikator der Veränderung der Mobilitätsbedürfnisse künftiger Kundengruppen und sind damit von großem Wert für die Weiterentwicklung von preisgünstigen und gleichzeitig flexiblen Verkehrsmitteln im Zeitalter der Digitalisierung.

In weiteren Projekten zur Überprüfung der Marktfähigkeit von autonomen Mobilitätskonzepten soll die Nutzerakzeptanz nicht nur auf dem Land sondern auch in der Stadt überprüft werden. Zudem sollen komplexere Verkehrsszenarien ausgewählt werden, um die Betriebsstabilität auch unter erschwerten Bedingungen zu testen. Nicht zuletzt soll in zukünftigen autonomen Projekten auch der Anschluss an die On-Demand-RidepoolingPlattform von ioki realisiert werden.

\section{Literatur}

acatech Studie (2016) Neue autoMobilität. Automatisierter Straßenverkehr der Zukunft. URL (22.03.2019) https://www.acatech.de/Publikation/neue-automobilitaet-automatisierter-strassenverkehr-der-zukunft/

ADAC (2017) Die Evolution der Mobilität. Eine Studie des Zukunftsinstituts im Auftrag des ADAC. URL (22.03.2019) https://www.zukunftsinstitut.de/fileadmin/user_upload/Publikationen/Auftragsstudien/ADAC_Mobilitaet2040_Zukunftsinstitut.pdf

Bad Birnbach (2018) Erster autonomer Bus in Deutschland. URL (26.03.2019) https://www.badbirnbach.de/presse/erster-autonomer-bus-in-deutschland

Berylls Strategy Advisors (2017) The Revolution of Urban Mobility. Studie zur urbanen Mobilität. URL (22.03.2019) https://www.berylls.com/wp-content/uploads/2018/01/20171216_Studie_Mobilitaet.pdf

Buffat M, Sommer H, Amacher M, Mohagheghi R, Beckmann J, Brügger A (2018) Individualisierung des ÖV-Angebots. Analyse der Auswirkungen der Individualisierung und weiterer angebots- und nachfragerelevanter Trends auf die zukünftige Ausgestaltung des ÖV-Angebots, Eidgenössisches Departement für Umwelt, Verkehr, Energie und Kommunikation UVEK

Conrad J (2016) Nachhaltige Mobilität im ländlichen Raum. Status quo und Entwicklungspotentiale bereits verfolgter Projekte in der Modellregion Schwarzwald-Baar-Heuberg, Global Studies Working Papers Institute of Geography, 34/2016, Eberhard Karls Universität Tübingen 
Deutsche Bahn (2019) Erstes autonomes Fahrzeug auf öffentlichen Straßen. URL (26.03.2019) https://www.deutschebahn.com/de/Digitalisierung/Smart-Mobility/Erstes-autonomes-Fahrzeug-auf-oeffentlichen-Strassen-3244104

Deutsche Bahn (2018) Faktenblatt Erste Autonome Buslinie Deutschlands. URL (26.03.2019) https://www.deutschebahn.com/resource/blob/259942/a0f34a180b5e6a0898d38c3eaff32570/ Faktenblatt-autonomer-Bus-data.pdf

Easymile (2019) EZ10 Autonomous Easymile Shuttle, URL (26.03.2019) https://easymile.com/solutions-easymile/ez10-autonomous-shuttle-easymile/

Heise A (2018) Pionierarbeit Hochautomatisiert fahrende Shuttles sind mittlerweile Realität - doch welche technischen Kriterien müssen sie erfüllen? TÜV Bus-Report 2018, Beilage zur Omnibusrevue 10/18, 10-11. URL (26.03.2019) https://www.omnibusrevue.de/fm/3333/OR\%2010\%20 2018_T\%C3\%9CV\%20Bus-Report.21917732.pdf

Herget M, Hunsicker F, Koch J, Chlond B, Minster C, Soylu T (2018) Ökologische und ökonomische Potenziale von Mobilitätskonzepten in Klein- und Mittelzentren sowie dem ländlichen Raum vor dem Hintergrund des demographischen Wandels

Horn B, Kiel T, von Lojewski H (2018) Nachhaltige städtische Mobilität für alle. Agenda für eine Verkehrswende aus kommunaler Sicht. Positionspapier des Deutschen Städtetages

Hunsicker F, Knie A, Lobenberg G, Lohrmann D, Meier U, Nordhoff S, Pfeiffer S (2017) Pilotbetrieb mit autonomen Shuttles auf dem Berliner EUREF-Campus, Internationales Verkehrswesen (69) $3,56-59$

ioki (2019): Autonomes Fahren. URL (26.03.2019) https://ioki.com/autonomes-fahren/

Münchner Kreis (2017) Mobilität. Erfüllung. System. Zur Zukunft der Mobilität 2025+. URL (22.03.2019) https://www.muenchner-kreis.de/download/zukunftsstudie7.pdf

Pavone M (2015) Autonomous Mobility-on-Demand Systems for Future Urban Mobility. In: Maurer M, Gerdes C, Lenz B, Winner H (Hrsg) Autonomes Fahren. Technische, rechtliche und gesellschaftliche Aspekte. Berlin/Heidelberg, S 399-416

Schaller B (2017) Unsustainable? The Growth of App-Based Ride Services and Traffic, Travel and the Future of New York City, Schaller Consulting. URL (22.03.2019) http://schallerconsult.com/ rideservices/unsustainable.pdf

VDV Das Magazin (2017) Titelstory: Autonomes Fahren als Zukunftsfrage. Ausgabe 01/2017. URL (22.03.2019) https://www.vdv-dasmagazin.de/story_01_2017-02-23_10-05-41.aspx

Open Access Dieses Kapitel wird unter der Creative Commons Namensnennung 4.0 International Lizenz (http://creativecommons.org/licenses/by/4.0/deed.de) veröffentlicht, welche die Nutzung, Vervielfältigung, Bearbeitung, Verbreitung und Wiedergabe in jeglichem Medium und Format erlaubt, sofern Sie den/die ursprünglichen Autor(en) und die Quelle ordnungsgemäß nennen, einen Link zur Creative Commons Lizenz beifügen und angeben, ob Änderungen vorgenommen wurden.

Die in diesem Kapitel enthaltenen Bilder und sonstiges Drittmaterial unterliegen ebenfalls der genannten Creative Commons Lizenz, sofern sich aus der Abbildungslegende nichts anderes ergibt. Sofern das betreffende Material nicht unter der genannten Creative Commons Lizenz steht und die betreffende Handlung nicht nach gesetzlichen Vorschriften erlaubt ist, ist für die oben aufgeführten Weiterverwendungen des Materials die Einwilligung des jeweiligen Rechteinhabers einzuholen.

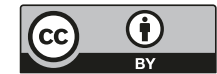

